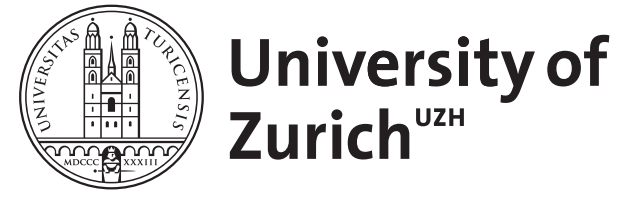

Zurich Open Repository and Archive

University of Zurich

Main Library

Strickhofstrasse 39

CH-8057 Zurich

www.zora.uzh.ch

Year: 2020

\title{
Spielarten der Rational-Choice-Theorie
}

Herfeld, Catherine

DOI: https://doi.org/10.1515/9783110673616-003

Posted at the Zurich Open Repository and Archive, University of Zurich

ZORA URL: https://doi.org/10.5167/uzh-191556

Book Section

Published Version

Originally published at:

Herfeld, Catherine (2020). Spielarten der Rational-Choice-Theorie. In: Tutić, Andreas. Rational choice.

Berlin: De Gruyter, 59-86.

DOI: https://doi.org/10.1515/9783110673616-003 


\section{I.3 Spielarten der Rational-Choice-Theorie}

\section{I.3.1 Einleitung}

Warum verhalten sich Menschen, wie sie es tun? Und warum sollten sie sich so (oder anders) verhalten? Antworten auf beide Fragen werden in den Verhaltens- und Sozialwissenschaften sowie der Philosophie von Handlungstheorien gegeben. Theorien rationalen Handelns fallen in eine spezifische Kategorie solcher Handlungstheorien. Sie beantworten die Frage, warum sich eine Person auf eine spezifische Art verhält bzw. verhalten sollte, indem sie auf die Rationalität der Person verweisen. Bei den meisten Varianten solcher Theorien wird das Handeln einer Person als Ergebnis eines rationalen Abwägungsprozesses verstanden.

Theorien rationalen Handels gehören zu den prominentesten Handlungstheorien der Sozialwissenschaften. Ihr Anwendungsbereich erstreckt sich über die Ökonomie, die Soziologie, die Politikwissenschaft und die Psychologie bis hin zur Philosophie. Damit sind sie zum gegenwärtigen Zeitpunkt aus den Sozialwissenschaften nicht wegzudenken. Gleichzeitig wird die Erklärung von Handlungen durch den Verweis auf die Rationalität der Akteure lange schon kontrovers diskutiert. Erstens erscheint es kontraintuitiv anzunehmen, dass unser Verhalten tatsächlich immer auf rationale Überlegungen zurückgeführt werden kann. Vielmehr kann menschlichem Handeln eine Vielzahl von Motivationen zugrunde liegen. Damit ist es nicht immer im klassischen Sinne rational. Zweitens sind die in den Theorien getroffenen Annahmen über individuelle kognitive Fähigkeiten als Voraussetzung für rationales Handeln oftmals nicht gegeben. Darüber hinaus unterliegen Handlungsüberlegungen von Individuen oft sogenannten „kognitiven Verzerrungen“, die einer rationalen Entscheidung entgegenwirken können. Diese Aspekte haben dazu geführt, dass Theorien rationalen Handelns immer wieder kritisiert und vielfach als empirisch nutzlos abgelehnt wurden.

In diesen Diskussionen wird oft von der einen Theorie rationalen Handelns für alle Sozialwissenschaften gesprochen, die auch als Received View bezeichnet wurde (z. B. Rosenberg 2015; Satz \& Ferejohn 1994). Dieser wurden drei Merkmale zugesprochen: (1) dass sie den methodologischen Status einer wissenschaftlichen Theorie hat, (2) dass sie mit einer Verpflichtung zum methodologischen Individualismus (MI) einhergeht und (3) dass sie sich auf ein Rationalitätsverständnis stützt, welches menschliches Verhalten als egoistisch beschreibt (Satz \& Ferejohn 1994). Als solche wird sie auf zwei Ebenen formuliert: auf der Ebene einer Handlungstheorie sowie auf einer formal-axiomatischen Ebene. 
Als Handlungstheorie basiert sie auf unserem alltagspsychologischen Verständnis von intentionalen Handlungserklärungen. Danach wird eine Handlung kausal mittels des Verweises auf Handlungsgründe bzw. mentale Zustände erklärt und vorausgesagt. Intentionale Handlungserklärungen basieren auf einer Definition von Handlung als Verhalten, welches durch Handlungsgründe motiviert und hervorgebracht wird, sowie auf alltagspsychologischen Begriffen wie beispielsweise „Bedürfnisse“ oder „Überzeugungen“, welche diese Gründe spezifizieren. ${ }^{1}$

Nach dem Received View wird die allgemein formulierte Handlungstheorie auf der zweiten Ebene formal-axiomatisch beschrieben. Handlungsgründe werden mittels technischer Begriffe wie dem Präferenzbegriff sowie dem Begriff der Überzeugungsgrade rekonstruiert. Beispielsweise beschreibt der Begriff einer Präferenzordnung formal-axiomatisch die Bedürfnisse eines Akteurs, die sein Handeln motivieren und dessen Ziele definieren. Als solche bekommt die RCT den Status einer allgemeinen wissenschaftlichen Theorie menschlichen Handelns, welche Handlungen mit Verweis auf intentionale Zustände erklärt oder voraussagt.

Dieser Received View hat oft zu Missverständnissen geführt. Schaut man sich die Anwendungsbereiche der Theorie an, so wird deutlich, dass es keine einheitliche Theorie rationalen Handelns gibt. Vielmehr kommen Theorien rationalen Handelns (hiernach: RCT) in verschiedenen Varianten vor. Man kann diese Varianten als verschiedene theoretische Ansätze auffassen, die jedoch eine gewisse Familienähnlichkeit aufweisen (z. B. Braun 2009; Diekmann \& Voss 2004; Herfeld 2013, 2020; Raub 2017). Aufgrund ihres breiten Anwendungsbereichs werden sie meist begrifflich und methodologisch an die Theoriegebäude aus den jeweiligen Disziplinen angepasst. In Diskussionen wurde die Unterscheidung dieser Varianten lange Zeit vernachlässigt, was oft zu einseitig formulierten und wenig konstruktiven Kritiken führte. In den letzten Jahren wurde vermehrt zwischen verschiedenen RC-Ansätzen unterschieden (z. B. Diekmann \& Voss 2004; Steele 2014; Thoma 2019). Dabei wurde jedoch meist nur auf eine Teilmenge aller RC-Varianten fokussiert, nämlich diejenigen Varianten, die ihren Ursprung in der Axiomatisierung des Nutzenmaximierungsprinzips durch John von Neumann und Oskar Morgenstern haben.

In diesem Kapitel werden verschiedene Spielarten der RCT mit Blick auf ihre Gemeinsamkeiten und Unterschieden vorgestellt (Abschnitt 2). Es wird deutlich, dass es weder nur eine RCT gibt, noch dass alle existierenden Varianten

1 Es wird debattiert, ob Handlungsgründe als Ursachen angesehen werden werden können oder nicht; siehe Davidson (1963) für ein zentrales Argument dafür, dass Handlungsgründe als Ursachen verstanden werden können. 
die drei Merkmale des Received Views erfüllen; es gibt zahlreiche Varianten der RCT, die mit diesem nur bedingt übereinstimmen. Es werden außerdem Erweiterungen der RCT skizziert (Abschnitt 3) und Minimalanforderungen identifiziert, die eine Handlungstheorie erfüllen sollte, um als Variante der RCT eingeordnet zu werden (Abschnitt 4).

\section{I.3.2 Die verschiedenen Spielarten der Theorie rationalen Entscheidens}

In der Literatur wird der Begriff „Theorie rationalen Entscheidens“ i.d. R. mit einer der folgenden fünf Varianten gleichgesetzt: mit (1) der Erwartungsnutzentheorie (EUT), (2) der subjektiven Erwartungsnutzentheorie (SEUT), (3) der Spieltheorie, (4) der Theorie offenbarter Präferenzen sowie mit (5) Gary Beckers Ökonomischem Ansatz. ${ }^{2}$ Sie werden im Folgenden mit Blick auf ihre Gemeinsamkeiten und Unterschiede skizziert.

\section{Die Erwartungsnutzentheorie}

Die EUT wird in den Sozialwissenschaften, der Philosophie sowie der Psychologie angewandt, um Entscheidungen unter Unsicherheit zu erklären, vorauszusagen oder vorzuschreiben. ${ }^{3}$ Ein zentrales Anwendungsproblem ist die individuelle Entscheidung. Wenn als Entscheidungstheorie angewandt, dann ist sie diejenige Variante, die am nächsten an den Received View herankommt. Bei der EUT handelt sich jedoch um eine axiomatische Messtheorie, die im zweiten Schritt auch empirisch interpretiert und damit auch als Entscheidungstheorie verwendet werden kann. In dem Fall geht man stets von dem Entscheidungsproblem eines Akteurs

\footnotetext{
2 Es gibt weitere Varianten der RCT, die hier nicht vorgestellt werden können. Die folgenden Varianten gelten insofern als zentral, als dass sie die Grundlage für andere Varianten sowie Erweiterungen bilden.

3 In der Literatur wird zwischen Sicherheit, Risiko, Unsicherheit und Unwissenheit unterschieden. In Situationen der Sicherheit wissen Akteure, welche Ereignisse als Konsequenz ihrer Handlungen realisiert werden. Grundsätzlich geht man davon aus, dass in Risikosituationen die objektiven Eintrittswahrscheinlichkeiten bekannt sind, wohingegen in Unsicherheitssituationen angenommen wird, dass sich ein Akteur subjektive Urteile über die Eintrittswahrscheinlichkeiten der Ereignisse bildet. Von Situationen der Unwissenheit spricht man, wenn man den verschiedenen Ereignissen keine Eintrittswahrscheinlichkeiten zuschreiben kann (für die Ursprünge der Unterscheidung siehe Knight (1921)).
} 
aus. Sein Entscheidungsverhalten ist das Ergebnis einer Auswahl zwischen verschiedenen Alternativen. Dabei wird das Verhalten mittels einer Präferenzrelation beschrieben. Genauer besteht die Theorie in ihrer Grundvariante aus drei Elementen: (1) einer binären Relation, (2) einer Anzahl an formalen Axiomen, die der binären Relation Struktur geben, indem sie als Bedingungen formuliert werden, welche die Relation erfüllen muss und (3) der Entscheidungsregel. Die bekannteste Regel ist die der Erwartungsnutzenmaximierung, nach dem ein Akteur immer diejenige Handlung wählt, die seinen erwarteten Nutzen maximiert (vgl. Kapitel II.1).

Ein wichtiger Unterschied zwischen der EUT und der Subjektiven Erwartungsnutzentheorie (SEUT) besteht darin, wie Präferenzen mittels dieser Elemente formal beschrieben werden. Eine zentrale Rolle spielt dabei das sogenannte Repräsentationstheorem und dessen Begründung. Als Repräsentationstheorem bezeichnet man den formalen Beweis, dass Akteurspräferenzen mittels einer Funktion dargestellt werden können, die den Nutzen der Akteure maximiert (siehe auch Thoma 2019: 66). Sowohl von Neumann und Morgenstern als Begründer der EUT als auch Leonard Savage als Begründer der SEUT gehen bei ihrem Repräsentationstheorem zunächst von einer binären Relation aus, welche bestimmte Eigenschaften erfüllt und damit als Ordnungsrelation klassifiziert werden kann. Diese wird im zweiten Schritt als Präferenzordnung interpretiert, wobei es je nach Anwendungskontext unterschiedliche Interpretationen davon gibt, was Präferenzen sind.

Welche Eigenschaften die Relation erfüllen muss, variiert je nach genauer Ausprägung der Theorie. Bei von Neumann und Morgenstern müssen u. a. das Transitivitätsaxiom, das Vollständigkeitsaxiom sowie das Axiom der Unabhängigkeit irrelevanter Handlungsalternativen erfüllt sein (vgl. Kapitel II.1). ${ }^{4}$ Alle drei Axiome sind zentral für die Herleitung der Nutzenfunktion und weil sie zusätzlich als plausible Eigenschaften von Präferenzen eines rationalen Akteurs interpretiert werden. Die Rationalität eines Akteurs manifestiert sich damit in einer Präferenzordnung, welche die Axiome erfüllt. Die Plausibilität der von Neumann-Morgenstern-Axiome wird jedoch kritisch diskutiert, da es fragwürdig ist, ob sie die tatsächlichen Präferenzen von Akteuren beschreiben. In empirischen

4 Neben den „Rationalitätsaxiomen“ müssen das Kontinuitätsaxiom, das Archimedische Axiom sowie das Reduktionsaxiom erfüllt sein. Auch das Kontinuitätsaxiom wurde als plausible Eigenschaft der Präferenzen eines rationalen Akteurs interpretiert. Es besagt, dass wenn ein rationaler Akteur die Wahl zwischen einer Lotterie $x$, bei der das beste Ergebnis mit einer bestimmten Wahrscheinlichkeit $p$ eintritt und das schlechteste Ergebnis mit einer bestimmten Wahrscheinlichkeit $1-p$ eintritt, und einer Lotterie $y$, bei der das mittlere Ergebnis mit Sicherheit eintritt, hat, sich immer eine Wahrscheinlichkeit $p$ finden lässt, bei der der Akteur indifferent ist bezüglich $x$ und $y$. 
Tests konnten sie vielfach nicht bestätigt werden. Darüber hinaus wurde argumentiert, dass auch intransitive Präferenzordnungen in manchen Fällen als vernünftig gelten können (siehe Thoma 2019: 84 ff.).

Von Neumann und Morgenstern beweisen mit ihrem Repräsentationstheorem die Existenz einer Nutzenfunktion. Diese besagt, dass ein Akteur eine Lotterie $x$ gegenüber einer anderen Lotterie $y$ genau dann vorzieht, wenn der Erwartungsnutzen von $x$ größer ist als der von $y$ (vgl. Kapitel II.1). Die so bewiesene Nutzenfunktion ist bis auf positiv-lineare Transformationen bestimmt. Damit ist sie nicht einzigartig in ihrer Eigenschaft die Präferenzen repräsentieren zu können. Vielmehr existieren weitere Nutzenfunktionen, die ebenfalls die Präferenzen repräsentieren. Die Nutzenfunktionen unterscheiden sich zwar mit Blick auf z. B. ihren Nullpunkt, sind aber strukturell identisch mit Blick auf das Verhältnis der Nutzenunterschiede einer bestimmten Präferenzordnung. Eine solche Nutzenfunktion bezeichnet man als kardinale Nutzenfunktion, die ermöglicht, Präferenzen zu messen. ${ }^{5}$

Die EUT in dieser allgemeinen Variante ist ohne weitere Spezifizierung, d. h. Interpretation der mathematischen Axiome und Begriffe wie „Relation“, „Nutzen“, „Präferenzen“, „Restriktionen“ etc., nicht anwendbar, um empirische Aussagen zu treffen. Im ersten Schritt gilt sie als rein formal-axiomatische Theorie, die unterschiedlich interpretiert und damit auf verschiedene, ggf. strukturell ähnliche Probleme angewandt werden kann. Sie hat daher nicht automatisch den Status einer wissenschaftlichen Theorie menschlichen Handelns. Vielmehr ist sie eine mathematische Theorie, die mit Blick auf ihre Interpretation und damit auf mögliche Anpassungen flexibel ist. Der Received View berücksichtigt dies nur insofern, als dass man hier die Interpretation der Theorie als Handlungstheorie bereits mitdenkt.

Genauer wird von der Interpretation einer formal-axiomatischen Theorie in zweierlei Hinsicht gesprochen. Erstens bezieht man sich oft auf die Interpretation der in der Theorie verwendeten Begriffe. Diese können unterschiedlich interpretiert werden. Mit Blick auf die EUT beispielsweise werden derzeit zwei Präferenzinterpretationen unterschieden: Präferenzen als beobachtbare Verhaltensmuster und als mentale Zustände (Guala 2019). Die Interpretation von Präferenzen hängt direkt mit der Interpretation des Nutzenbegriffs zusammen. Lange ging man in den Wirtschaftswissenschaften vom Nutzen als psychologische Entität aus, die man mit entsprechenden Instrumenten messen kann (z. B.

5 Im Gegensatz zur kardinalen Nutzenfunktion lässt eine ordinale Nutzenfunktion keine Aussagen über das Ausmaß der Nutzenunterschiede, sondern alleine über die Ordnung selber zu. Sie ist nur bis auf beliebige positiv monotone Transformationen bestimmt. 
Edgeworth 1881; Jevons [1871] 1888). In der SEUT nimmt man an, dass der Nutzen uns etwas über die Intensität der Präferenzen eines Akteurs sagt. Ebenso werden Wahrscheinlichkeiten als subjektive Überzeugungsgrade eines Akteurs interpretiert. Daneben gibt es aber auch die Interpretation von Nutzen als eine rein theoretische Darstellung der Präferenzordnung.

Zweitens wird von der Interpretation einer axiomatischen Theorie gesprochen, wenn es um ihre Anwendung geht. Einerseits wird sie als normative Theorie inter pretiert, die als Standard verwendet wird, um einem Akteur vorzuschreiben, was in einer bestimmten Situation die rationale Entscheidung wäre. Sie wird auch als normative Theorie verwendet, um ex post zu beurteilen, ob eine bereits getroffene Entscheidung rational oder irrational war. Andererseits wird sie auch als empiri. sche Theorie interpretiert, um tatsächlich getroffene Entscheidungen ex post zu er. klären bzw. ex ante vorauszusagen.

Dass für die Anwendung der Theorie eine Interpretation erforderlich ist, wird in der erklärenden Soziologie durch die Unterscheidung zwischen einer engen und einer weiten Variante der RCT weiter konkretisiert (Opp 1999). Die enge Variante basiert neben dem Nutzenmaximierungsprinzip auf Zusatzan. nahmen, die sich auf den Informationsstand und Akteursziele, auf die Eigen. schaften bzw. die Interpretation der Präferenzen sowie der Handlungsoptionen im jeweiligen Anwendungskontext beziehen. Es wird angenommen, dass $\mathrm{Ak}$. teure vollständige Informationen haben und Präferenzen stabil sind, d. h. sich im Zeitablauf nicht ändern. Damit werden Verhaltensänderungen allein auf Veränderungen in den verfügbaren Handlungsoptionen bzw. Restriktionen $\mathrm{z}_{\mathbf{L}}$. rückgeführt. Auch wird oft die oben genannte Egoismus-Annahme getroffen, nämlich dass Akteure ihre Handlungsalternativen auf Basis ihrer persönlichen Interessen ordnen und ihren materiellen bzw. monetären Nutzen maximieren (Rössel \& Weingärtner 2016: 8 ff.). ${ }^{6}$ Ein letztes Element der engen Variante stellen die den Akteuren zur Verfügung stehenden materiellen oder immateriellen Ressourcen bzw. Restriktionen dar, welche Handlungsmöglichkeiten darin beschränken, die eigenen Ziele zu erreichen. In der engen Variante werden alleine monetäre Beschränkungen, wir beispielsweise das Einkommen, berücksichtigt.

Je nachdem wie der Nutzen- sowie der Präferenzbegriff formalisiert und interpretiert werden, können auch nicht-egoistische Motive wie beispielsweise moralische, sozial orientierte und emotional motivierte Handlungen sowie jedwede Art von materiellen und immateriellen Handlungsrestriktionen abgebildet werden. Man spricht dabei von der weiten Variante (Opp 1999). Akteure sind bei dieser Variante nur unvollständig informiert und Präferenzen können sich im Zeitablauf ändern.

6 Opp (1999) differenziert nicht zwischen verschiedenen Varianten der RCT. 
Die weiten Varianten der EUT machen keine Einschränkungen bezüglich der zugelassenen Präferenzen. Da man die tatsächlichen Präferenzen der Akteure empirisch oftmals nicht eindeutig ermitteln kann und man demnach vom Verhalten der Akteure auf ihre Präferenzen schließt (siehe Abschnitt 2), wurde bei der Verwendung der weiten Variante der EUT als erklärende Handlungstheorie oft der Vorwurf der tautologischen bzw. zirkulären Erklärung formuliert. Man schließt von beobachtbarem Verhalten auf unbeobachtbare Präferenzen, um diese dann als Ursache für das Verhalten zu identifizieren und damit das Verhalten zu erklären. Jedoch würden Vertreter wie beispielsweise Opp (2019: 99) dem Zirkularitätsvorwurf mit dem Argument begegnen, dass Präferenzen immer nur ex ante bzw. unabhängig vom Verhalten und von Restriktionen bestimmt werden.

In ihren engen und weiten Varianten wird die EUT vielfach als Handlungstheorie und in Kombination mit dem methodologischen Individualismus (MI) verteidigt. Sie bietet eine von verschiedenen Entscheidungsregeln für Entscheidungssituationen unter Unsicherheit. Es stellt sich jedoch die Frage, inwiefern es sich um eine wissenschaftliche Theorie im traditionellen Sinne handelt, die tatsächlich eine Gesetzmäßigkeit des menschlichen Handelns beschreibt so wie es der Received View annimmt. Die EUT hat nicht immer den Status einer Entscheidungstheorie. In den Wirtschaftswissenschaften, in der erklärenden Soziologie sowie in der positiven Politikwissenschaft dient sie meist als theoretischer Ausgangspunkt, um soziale Phänomene unter Rückgriff auf individuelles Handeln zu erklären und vorauszusagen. Als Explananda gelten jedoch soziale bzw. kollektive Phänomene, wie Wahlausgänge, Preisänderungen, gesellschaftlicher Wandel, Wirtschaftswachstum, die Auswirkungen finanzpolitischer Maßnahmen auf Marktverhalten, und Makroeffekte der sozialen Interaktion zwischen Individuen, wie beispielsweise Kriminalitätsraten, Heiratsmuster, die Entstehung sozialer Ordnung oder Marktstrukturen (Diekmann \& Voss 2018). Erklärungen dieser Phänomene werden mit Verweisen auf Akteurshandlungen, die Art der Interaktionen zwischen den Akteuren sowie auf die soziale Situation gegeben, innerhalb der die Handlungen stattfinden. Dieser Strategie liegt meist eine Verpflichtung zum MI zugrunde. Das Postulat des MI besagt, dass soziale Phänomene immer mit Verweis auf individuelles Verhalten erklärt werden sollen (Satz \& Ferejohn 1994; Udehn 2001). ${ }^{7}$ Es handelt sich dabei um ein methodologisches Prinzip, welches auf der Metaebene vorschreibt, wie soziale Phänomene mittels Theorien oder Modellen erklärt werden sollen. Das Postulat hat selber keinen Wahrheitswert.

\footnotetext{
7 Das Postulat des methodologischen Individualismus kommt in zahlreichen Varianten vor, auf die hier nicht ausreichend eingegangen werden kann (Udehn 2001).
} 
Die Varianten der RCT können für solche Erklärungen herangezogen werden; angewandt als wissenschaftliche Theorien können sie wahr oder falsch sein (Mantzavinos 2009). Die EUT wird für solche Erklärungen jedoch oft für die Formulierung von Annahmen über individuelles Handeln herangezogen, welches in einen sozialen Kontext eingebettet ist. Als solche ist sie integrativer Bestandteil einer übergeordneten Theorie, was in der Soziologie eine spezifische Sozialtheorie (z. B. die Marx'sche Theorie; siehe Elsters Analytischen Marxismus (1985)) oder in den Wirtschaftswissenschaften eine allgemeine Markttheorie (z. B. die allgemeine Gleichgewichtstheorie) sein kann. Als einer von mehreren Bestandteilen eines übergeordneten theoretischen Ansatzes verliert sie damit ihren methodologischen Status als wissenschaftliche Handlungstheorie. Anders als vom Received View vorausgesetzt, wird sie auf eine Verhaltensannahme reduziert, die neben anderen Annahmen die Anwendungsbedingungen der übergeordneten Theorie fixieren. Als solche muss sie anderen epistemischen Anforderungen genügen als eine wissenschaftliche Theorie.

Darüber hinaus bekennen sich die Sozialwissenschaften zwar oft und explizit zum MI (Sugden 2016: 1379). In der konkreten Praxis bleibt es jedoch in vielen Teilbereichen bei einem rhetorischen Bekenntnis. Beispielsweise werden in den Wirtschaftswissenschaften unter dem Begriff des „Akteurs“ vielfach kollektive Akteure wie beispielsweise Organisationen, Unternehmen oder Staaten gefasst. Diese Strategie führt die Erklärung von kollektiven Akteuren gerade nicht zurück auf die Ebene des Individuums. In ökonomischen Modellen ist meist von repräsentativen Akteuren die Rede, die idealtypische Eigenschaften wie beispielsweise die eines typischen Konsumenten oder Produzenten erfüllen. Diese Strategie, obwohl näher an der Forderung des MI, ignoriert die Eigenschaften des Individuums und dessen Verhaltens sowie die Heterogenität von Individuen. Je nachdem wie man die Forderung des MI schlussendlich definiert, stellt sich daher die Frage, inwiefern solche Modellierungen tatsächlich mit Verweis auf individuelle Handlungen erklären.

Die EUT spielt auch in formalen Modellen sowie in computergestützten Verfahren ebenso wie in der sozialwissenschaftlichen Theoriebildung eine zentrale Rolle (Diekmann \& Voss 2004; Opp 2014; Hedström \& Ylikoski 2010). In der Verhaltens- und Experimentalökonomik kommt sie als normative Theorie und damit als benchmark zum Einsatz. Als deduktiver Ansatz erlaubt sie Theoreme abzuleiten, die man als Aussagen über Verhalten (meist) in hypothetischen Situationen interpretieren kann. ${ }^{8}$ Wenn ihre Aussagen vom tatsächlichen Verhalten

8 Es kann durchaus sein, dass sich Akteure im Experiment tatsächlich gemäß den Voraussagen der EUT verhalten. 
abweichen, dann hilft sie, das experimentelle Design sowie die Daten zu strukturieren. Sie trägt zur Theoriebildung bei, indem sie den Wissenschaftler bzw. der Wissenschaftlerin zwingt, die Abweichungen des tatsächlichen Verhaltens von theoretischen Voraussagen zu erklären (Schotter 2006). Andere verstehen die axiomatische Theorie selber als Modell und verwenden sie gemeinsam mit Kontexthypothesen und Aggregationsregeln dafür, um soziale Phänomene - soziale Prozesse und kollektive Effekte als Ergebnis sozialer Interaktion - zu modellieren (vgl. Kapitel I.2; Diekmann \& Voss 2004: 21). Man kann die EUT deshalb als Teil des sozialwissenschaftlichen Werkzeugkastens verstehen, der für verschiedene Probleme zur Anwendung kommt.

\section{Leonard Savages subjektive Erwartungsnutzentheorie}

Um Entscheidungen unter Unsicherheit abzubilden, gingen von Neumann und Morgenstern von objektiven und bekannten Wahrscheinlichkeiten aus, die jedem Ereignis zugeordnet sind. Mit seiner SEUT erweiterte der Statistiker und Mathematiker Leonard Savage (1954) die EUT (vgl. Kapitel II.1). Savage ging davon aus, dass Akteure subjektive Überzeugungsgrade bezüglich der möglichen Umweltzustände im Lichte gegebener Information bilden und Präferenzen bezüglich aller möglichen Handlungsalternativen sowie Ereignisse haben. In Savages Variante müssen Präferenzen ebenfalls eine Anzahl von Axiomen erfüllen. Die subjektiven Überzeugungsgrade müssen den Wahrscheinlichkeitsaxiomen genügen und der Bayesianischen Wahrscheinlichkeitsinterpretation folgen (Diekmann \& Voss 2004). ${ }^{9}$ So können eindeutige Nutzenwerte und subjektive Wahrscheinlichkeiten identifiziert werden.

Die SEUT stellt, ebenso wie die EUT, eine Formalisierung des Rationalitätsprinzips dar. Der rationale Akteur maximiert seinen subjektiven Erwartungsnutzen. Die Nutzenfunktion wird auch aus einer Theorie rationaler Präferenzen abgeleitet. Nach Savage müssen rationale Präferenzen vier Axiome erfüllen: Transitivität, Vollständigkeit, das Sure-Thing-Prinzip sowie das Wahrscheinlichkeitsprinzip. Das sogenannte Sure-Thing-Prinzip steht insofern in enger Beziehung zum Unabhängigkeitsaxiom als dass es letzteres impliziert - dies allerdings unter der Annahme, dass die Wahrscheinlichkeiten bekannt sind (vgl. Kapitel II.1).

\footnotetext{
9 Da die Wahrscheinlichkeiten bei der EUT bekannt sind, wird sie oftmals als eine Entscheidungstheorie in Risikosituationen bezeichnet, wohingegen die SEUT oft als Entscheidungstheorie unter Unsicherheit eingestuft wird.
} 
Erfüllt eine Präferenzordnung die Axiome, dann kann man ein Repräsentationstheorem beweisen, welches die Präferenzordnung funktional repräsentiert. Diese Formulierung ist im ersten Schritt auch hier rein formal-mathematisch. Erst im zweiten Schritt werden die Axiome als Eigenschaften von Präferenzen eines rationalen Akteurs interpretiert und der funktionale Zusammenhang als Nutzenfunktion. Die Interpretation der Axiome wird ebenfalls oft auf Basis von Plausibilitätsüberlegungen gerechtfertigt. Beispielsweise erscheint es nicht plausibel, dass ein rationaler Akteur zirkuläre Präferenzen hat. Durch sogenannte Money Pump- bzw. Dutch Book-Argumente wird diese Intuition explizit gemacht. ${ }^{10}$ Die Interpretation der Axiome stellt sicher, dass eine Handlung einer anderen Handlung genau dann vorgezogen wird, wenn der subjektive Erwartungsnutzen dieser Handlung größer ist als derjenige der anderen Handlung.

Diekmann \& Voss (2004: 17) argumentieren, dass die formal-axiomatischen Varianten der RCT in den Sozialwissenschaften einerseits als zu schwach und andererseits als zu stark eingestuft werden; zu schwach, da es sich bei der EUT sowie der SEUT grundsätzlich um Messtheorien handelt. Die messtheoretische Dimension wird oftmals vernachlässigt. Genauer ist die Nutzenfunktion im Sinne einer Messtheorie nichts anderes als eine Abbildung der Akteurspräferenzen in die Menge der reellen Zahlen. Wie angedeutet, geht die EUT in ihrer Minimalform davon aus, dass Handlungsalternativen auf Basis von Präferenzen in eine Reihenfolge gebracht werden. Die Reihenfolge ergibt sich aus den Axiomen der Theorie, die von der Ordnungsrelation erfüllt werden müssen. Aus diesen Axiomen wird die Nutzenfunktion in Form eines Repräsentationstheorems deduktiv hergeleitet. Damit erhält sie die Präferenzordnung des Akteurs aufrecht. Die EUT hat also den epistemischen Status einer Messtheorie von Präferenzen. Die formal-axiomatischen Varianten werden jedoch oft auch als zu stark eingestuft, nämlich dann, wenn sie als universelle Entscheidungstheorien angesehen werden; sie werden als erklärende und voraussagende Theorien überschätzt. Obwohl sie auch als rein mathematische Theorien ihren Nutzen haben, so sind die empirische Überprüfbarkeit sowie Stützung der Rationalitätsaxiome doch essentiell, um sie auf menschliches Verhalten anwenden zu

10 Sogenannte Money-Pump- sowie Dutch Book-Argumente (Ramsey 1928; Davidson et al. 1955) basieren auf einem Gedankenexperiment, welches das Transitivitätsaxiom als notwendige Bedingung für rationale Präferenzen plausibel machen soll. Money-Pump-Argumente zeigen, dass ein Akteur mit zirkulären Präferenzen auf dem Markt ausgebeutet werden kann. Jedoch basiert auch diese Art von Argumenten wiederum auf Annahmen, die nicht ohne Weiteres akzeptiert werden müssen (Machina 1989). 
können. Dies ist allerdings nicht trivial, wie im Falle der SEUT auch Paradoxa wie das Ellsberg-Paradox sowie, im Falle der EUT, das Allais-Paradox zeigen (vgl. Kapitel IV.1). ${ }^{11}$

\section{Die Spieltheorie}

Meist geht es in den Sozialwissenschaften weniger um individuelles Verhalten als vielmehr um die Interaktion verschiedener Akteure in unterschiedlichen sozialen Situationen und die durch diese Interaktionen entstehenden Muster. Weder die EUT noch die SEUT sind ohne Weiteres auf solche Situationen anwendbar. Die Schwierigkeit ergibt sich daraus, dass bei sozialen Interaktionen die Realisierung der Ereignisse immer auch vom Verhalten anderer Akteure abhängig ist. Um die Interdependenz von Handlungen theoretisch abbilden zu können, greifen die Sozialwissenschaften auf die Spieltheorie zurück. In der EUT sowie der SEUT sind die Eintrittswahrscheinlichkeiten der Umweltzustände exogen gegeben. In der Spieltheorie wird diese Annahme infrage gestellt.

Die Spieltheorie modelliert Situationen sozialer Interaktion, wie beispielsweise des Konflikts bzw. der Kooperation, als strategische Interaktion zwischen rationalen Akteuren (vgl. Kapitel II.2; Kapitel II.3). Die Bezeichnung „strategisch" verweist auf die Tatsache, dass Akteure die Handlungen Dritter insofern mit in ihre Entscheidungsüberlegungen aufnehmen als dass diese Auswirkungen auf das eigene Entscheidungsergebnis haben können (Steele 2014: 191). In der Modellierung wird eine soziale Situation als Spiel aufgefasst. Das Spiel wird durch die Spezifizierung aller Akteure und ihrer Handlungsoptionen bzw. Strategien beschrieben. Jede mögliche Kombination von Handlungsoptionen kann zu unterschiedlichen Ereignissen führen, bezüglich derer die Akteure Präferenzen haben. Es werden Annahmen über die Präferenzeigenschaften und den Informationsstand der Spieler gemacht. Präferenzen werden auch in der Spieltheorie durch eine Nutzenfunktion beschrieben, die verschiedene Formen annehmen und damit unterschiedliche Motive (z. B. egoistische, prosoziale und altruistische Motive) repräsentieren kann.

Um die Interdependenz von Handlungen zu modellieren, geht die Spieltheorie davon aus, dass die Eintrittswahrscheinlichkeiten gewisser Umweltzustände nicht mehr exogen gegeben sind, sondern in Abhängigkeit des Verhaltens aller anderer Akteure endogen bestimmt werden. Es wird angenommen, dass Akteure

11 Das Allais-Paradox (Allais 1953) sowie das Ellsberg-Paradox (Ellsberg 1961) zeigen auf, dass tatsächliches Akteursverhalten oftmals nicht den Rationalitätsaxiomen entspricht. 
im Zusammenhang mit ihren Entscheidungen auch Erwartungen bezüglich des Verhaltens anderer Akteure bilden und ihre eigenen Erwartungen von diesen abhängig machen. Diese Annahme impliziert, dass ein Akteur Erwartungen über das Verhalten anderer Akteure bildet, die selber wiederum Erwartungen bilden über das Verhalten des Akteurs, der wiederum Erwartungen über das Verhalten dieser Akteure bildet, ad infinitum. Dieser infinite Regress muss zu einem bestimmten Zeitpunkt abgebrochen werden, damit eine Entscheidung getroffen werden und es zur Handlung kommen kann. Gleichzeitig macht dieser Prozess die tatsächlich resultierenden Handlungen nicht genau vorhersagbar. Daher werden Lösungskonzepte formuliert, die spezifizieren, wie sich die Spieler am Spielende entscheiden. Diese sind oftmals in Form eines „Gleichgewichts“ formuliert, was intuitiv plausibel erscheint. Man erwartet, dass rationale Spieler am Ende eine Situation erreichen möchten, in der niemand einen Anreiz hat, das eigene Verhalten zu ändern, d. h. eine Situation zu erreichen, die stabil ist - daher der Begriff des „Gleichgewichts“.

Das bekannteste Lösungskonzept ist das von dem Mathematiker John Nash formulierte Nash-Gleichgewicht (Nash 1950). Das Nash-Gleichgewicht ist ein Begriff aus der nicht-kooperativen Spieltheorie, der eine Kombination von Strategien bezeichnet, welche alle rationalen Spieler so wählen, dass der einzelne Spieler keinen Anreiz hat, die eigene Strategie zu wechseln, falls alle anderen Spieler ihre Strategie auch nicht wechseln; die Strategie des Spielers gilt als die beste Antwort (best response) auf die Strategiewahl der anderen Spieler. Im Gleichgewicht erzielt der einzelne Akteur den höchsten Erwartungsnutzen gegeben der Handlungen aller anderen Akteure. ${ }^{12}$ Neben den Rationalitätsaxiomer der SEUT bzw. der EUT werden weitere Annahmen gemacht, um sicherzustellen, dass Spieler tatsächlich die Strategie wählen, die zum Nash-Gleichgewicht führt. Eine zentrale Annahme ist die common knowledge-Annahme (Vanderschraaf \& Sillari 2014). Die Spieler haben vollständige Informationen bezüglich des Aufbaus des Spiels, ihrer eigenen Präferenzen sowie der Präferenzen und des erwarteten Nutzens ihrer Mitspieler. Die Annahme setzt ebenfalls voraus, dass alle Spieler denselben Rationalitätsannahmen folgen.

Die common knowledge-Annahme findet sich in ähnlicher Form in anderen Varianten der RCT wieder. Grundsätzlich wird in Entscheidungstheorien unter S i cherheit, wie beispielsweise in der ordinalen Nutzentheorie sowie dem Ökonom i schen Ansatz von Gary Becker, vollständige Information auf Seiten der Akteure

12 Ein Nash-Gleichgewicht bringt nicht notwendigerweise das auf der kollektiven Ebene bes te Ergebnis hervor, wie beispielsweise das Gefangenendilemma zeigt. 
angenommen. ${ }^{13}$ Solche Annahmen wurden in der Literatur kritisch diskutiert (vgl. Kapitel II.4). ${ }^{14}$ Die common knowledge-Annahme ist nicht minder kontrovers. Es wird einerseits argumentiert, dass die Annahme die rationalen Spieler notwendigerweise zum Nash-Gleichgewicht führt. In der evolutionären Spieltheorie wird andererseits argumentiert, dass Spieler grundsätzlich nicht rational handeln, sondern dass durch Adaption und Selektion diejenigen Spieler in einem spezifischen Kontext favorisiert werden, die so handeln als ob sie rational wären. Damit erreichen sie zwar das Nash-Gleichgewicht, jedoch nicht durch rationale Überlegungen (Steele 2014: 195). Nash-Gleichgewichte werden also nur teilweise als das Ergebnis rationaler Überlegungen und unter der Bedingung der vollständigen Information angesehen.

Darüber hinaus ist zwar das Nash-Gleichgewicht eine notwendige aber keine hinreichende Bedingung für ein Lösungskonzept. Manche Spiele haben mehrere Nash-Gleichgewichte; Koordinationsspiele sind ein Beispiel. Für das sogenannte Selektionsproblem, d. h. die Wahl des am Ende realisierten NashGleichgewichts, wurden unterschiedliche Lösungen gefunden. Diese gehen insofern vielfach über die Spieltheorie hinaus als dass sie nicht von der formalen Spielstruktur fixiert werden. Beispielsweise hat Thomas Schelling (1960) den Begriff des Fokalpunkts als eine Lösungsmöglichkeit für das Selektionsproblem eingeführt. ${ }^{15}$ Nach Schelling hängt die Entscheidung von der Salienz der Alternativen ab. Die Salienz einer Alternative hängt von der Beurteilung ihrer Eigenschaften durch die Akteure auf Basis von kulturellen Hintergründen, Erfahrungen und Wissensmustern ab. Eine Handlung wird auf dieser Basis als Fokalhandlung und damit als die beste fixiert. Daneben wurde argumentiert, dass soziale Normen die Gleichgewichtswahl vorschreiben (Bicchieri 2006). Bei diesen Vorschlägen stellt sich jedoch die Frage, in welchem Sinne sie mit dem Grundgedanken der rationalen Akteure zu vereinen sind.

Bei der Spieltheorie handelte es sich ursprünglich um eine rein mathematische Theorie. Zwar hat von Neumann zunächst allein (1928) und dann gemeinsam mit

13 Die ordinale Nutzentheorie wird i.d.R. im Rahmen einer Markttheorie verwendet, um das Verhalten von ökonomischen Akteuren zu beschreiben. Die neoklassische Markttheorie fordert, dass vollkommene Transparenz bezüglich der Daten und Sachverhalte auf Seiten aller Akteure besteht. Nur wenn keine Informations- bzw. Transaktionskosten anfallen, führt die Interaktion der Marktteilnehmer zur Markträumung.

14 Ein wesentlicher Unterschied zwischen der Annahme vollständiger Information und der common knowledge-Annahme ist, dass bei der letzteren die Akteure zusätzlich Informationen über das Wissen aller anderen Akteure haben.

15 Schelling hat primär Koordinationsspiele untersucht und zu Illustrationszwecken weitestgehend informelle, wie er selber sagte „unwissenschaftliche“, Experimente durchgeführt. Für eine experimentalökonomische Analyse siehe Metha et al. (1994). 
Morgenstern (von Neumann \& Morgenstern [1944] 1953) verschiedene Spielformen und Entscheidungsregeln in der nicht-kooperativen Spieltheorie formuliert und damit die Grundlagen für die heutige Spieltheorie gelegt. Darüber hinaus wurde durch die Zusammenarbeit mit Morgenstern die Erwartung geschürt, dass die Spieltheorie direkten Anwendungsbezug in den Wirtschaftswissenschaften hat. Lange konnte dies jedoch bis auf wenige Ausnahmen nicht realisiert werden, $\mathrm{u}$. a. weil sie mathematisch $\mathrm{zu}$ anspruchsvoll war. Bis heute gehören Teilbereiche der Spieltheorie zur Mathematik. Erst im zweiten Schritt, nämlich wenn die abstrakten Begriffe wie die Ordnungsrelation, die Axiome, die Theoreme und Spielstrukturen eine entsprechende Interpretation erhalten, kann man auch hier von einer sozialwissenschaftlichen Theorie sprechen. Als solche wird die Spieltheorie ebenso wie die EUT und die SEUT sowohl als empirische als auch als normative Theorie interpretiert. Ziel ist am Ende zu erklären, vorauszusagen oder vorzuschreiben, welche Strategien die einzelnen Akteure wählen bzw. wählen sollen und welche Gleichgewichte dabei realisiert werden bzw. realisiert werden sollten.

\section{Gary Beckers Ökonomischer Ansatz}

Gerade in den Sozialwissenschaften wird die Bezeichnung der „Rational Choice Theory" vor allem in Verbindung mit Gary Beckers Ökonomischem Ansatz für menschliches Verhalten gebracht. Der Chicagoer Ökonom hat den Begriff der „Theorie rationalen Entscheidens“ nicht eingeführt. Trotzdem wurde der Begriff durch die Bezeichnung des Ökonomischen Ansatzes als „Rational-Choice-Ansatz“ nicht nur ein populärer Platzhalter für verschiedene Ansätze in der Entscheidungs- und Spieltheorie, sondern auch für die marginalistische Nutzentheorie (Vriend 1996). Die breite Begriffsanwendung führte oft zu einer Gleichsetzung von konzeptionell verschiedenen Ansätzen - beispielsweise der EUT und dem Ökonomischen Ansatz - und damit zu Missverständnissen.

Ein wesentlicher Unterschied zwischen dem Ökonomischen Ansatz und der EUT sowie der SEUT ist, dass es sich bei letzteren um axiomatische Theorien handelt, wohingegen Becker die axiomatische Methode in der ökonomischen Theorie ablehnt. Becker nimmt auch das Nutzenmaximierungsprinzip als theoretischen Ausgangspunkt. Er geht jedoch in seinem Ansatz nicht von einer Anzahl an Rationalitätsaxiomen aus, wie es in der EUT sowie die SEUT der Fall ist. Vielmehr wird der Ansatz in der marginalen Nutzentheorie verortet, in der die Nutzenfunktion ad hoc postuliert und unter Nebenbedingungen maximiert wird. Becker (1976: 5) macht drei Grundannahmen: „The combined assumptions of [1] maximizing behavior, [2] market equilibrium, and [3] stable preferences used relentlessly 
and unflinchingly“. Damit macht Becker zwar Verhaltensannahmen, die sich in den Eigenschaften der Nutzenfunktion widerspiegeln. Diese haben jedoch nicht den Status von formalen Axiomen, die zur Herleitung eines Repräsentationstheorems und damit zur formalen Begründung der Nutzenfunktion dienen. Obwohl Beckers Ansatz vom Received View nicht erfasst wird, ist dieser zweifellos eine zentrale Variante der RCT. Eine formal-axiomatische Formulierung einer alltagspsychologischen Handlungstheorie ist also keine Minimalanforderung an eine RCT.

Der Begriff des rationalen Handelns wird bei Becker auch als Nutzenmaximierung interpretiert. Ebenso wie die EUT sowie die SEUT geht Becker von dem Entscheidungsproblem eines Akteurs aus. Er macht jedoch deutlich, dass dieses ein Allokationsproblem im klassisch ökonomischen Sinne ist. Es geht darum, eine begrenzte Menge an Ressourcen vor dem Hintergrund gewisser Restriktionen, wie beispielsweise einem begrenzten Budget oder einem begrenzten Zeithorizont, und bestimmter Opportunitätskosten, die jede Entscheidung mit sich bringt, optimal für die eigenen Zwecke einzusetzen. Das Maximierungsprinzip gibt eine Lösung für das Allokationsproblem vor. Mathematisch wird die Lösung durch die Maximierung der Nutzenfunktion unter gegebenen Nebenbedingungen gefunden; letztere erfasst die externen Beschränkungen des Akteurs. Wie auch in der Spieltheorie geht es darum, die Lösung als Gleichgewicht darzustellen. Beckers Prinzip des Marktgleichgewichts setzt die Existenz eines Marktes voraus, welcher die Handlungen der Akteure in mehr oder weniger effizienter Weise koordiniert. Akteure verfolgen keine Strategien im Sinne der Spieltheorie. Vielmehr nehmen Preise und andere Marktinstrumente (beispielsweise das Einkommen) die Funktion der Sicherung einer effizienten Ressourcenallokation ein, u. a. indem sie den Handlungsspielraum des Akteurs beschränken. Das Gleichgewicht kommt dadurch zustande, dass Akteure auf einem Markt innerhalb extern gegebener Rahmenbedingungen handeln und nicht dadurch, dass, wie in der Spieltheorie angenommen, der einzelne Akteur sich am Verhalten anderer Akteure orientiert und dann die beste Strategie wählt.

Becker nimmt auch an, dass sich Präferenzen weder über die Zeit hinweg fundamental verändern noch, dass sie sich über Akteursgruppen grundsätzlich voneinander unterscheiden. Dies gilt auch für Akteure, die sich mit Blick auf charakteristische Eigenschaften (z. B. bzgl. Geschlecht, Einkommen, Ethnie, Kultur etc.) unterscheiden. Bei Beckers Präferenzen handelt es sich jedoch nicht um Präferenzen über gewöhnliche Konsumgüter oder Handlungsergebnisse, wie es in der EUT und der SEUT formuliert werden kann. Vielmehr hat ein Akteur nur Präferenzen bezüglich fundamentaler Grundgüter, sogenannter „fundamental aspects of life“ (Becker 1976: 5). Bei diesen Grundgütern handelt es sich um universelle Grundbedürfnisse wie beispielsweise Reichtum, Gesundheit, 
Prestige, Nächstenliebe und Gemeinschaftsgefühl. Diese werden mittels Konsumguiter vom Akteur selber produziert. Becker nimmt an, dass Akteure für die Produktion solcher Güter Zeit, verschiedene Inputgüter und Dienstleistungen verwenden. Letztere werden am Markt angeboten und nachgefragt. Die Grundgüter stehen nicht in einer eindeutigen und gleichbleibenden Beziehung zu Produktionsgütern und Dienstleistungen (Becker 1976: 5ff.). Das Grundgut „Prestige“ kann mittels verschiedener Inputgüter produziert werden. Diese werden auf dem Markt nachgefragt. Ihre Nachfrage ist abhängig von ihren Preisen und dem Einkommen der Akteure. Daher kann sie variieren.

Es gibt $n$ Grundgüter (Index $j$ ) und $m$ Haushalte (Index $i$ ), die sich alleine mit Blick auf die Produktion und damit auf die Inputgüter unterscheiden, nicht mit Blick auf die Nutzenfunktion. Formal werden alleine die fundamentalen Grundgüter $Z_{j, i}$ und nicht die Produktionsgüter in die Nutzenfunktion aufgenommen und damit erweitert. Beispielsweise maximiert ein Haushalt $i$ die Gewinne, die er aus der Produktion von „Prestige“ $Z_{1, i}$ ziehen kann. Die Nutzenfunktion ist selbst eine abdiskontierte Summe an Funktionen; die Grundgüter sind auf einen Zeitraum fixiert und die Diskontrate wird durch die Zeitpräferenz eines Haushalts festgelegt.

$$
U_{i}=u\left(Z_{1, i}, \ldots, Z_{n, i}\right) \text { mit } i=1, \ldots, m
$$

$Z_{j, i}$ bezeichnet die Menge des Grundguts $j$, die gemäß der Produktionsfunktion des $i$-ten Haushalts $f_{j, i}$ produziert wird. Der Haushalt bezieht Nutzen aus den produzierten Gütern auf Basis von Inputfaktoren. Beispielsweise ist das Kochen mit Personen ein möglicher Inputfaktor, um das Grundgut „Gemeinschaftsgefühl“ zu produzieren. Insofern dienen Marktgüter und Dienstleistungen nur als Inputfaktoren im Produktionsprozess. Die Produktionsfunktion kann für jeden Haushalt unterschiedlich sein. Ganz allgemein hat sie folgende Form:

$$
Z_{j, i}=f_{j, i}\left(x_{i}, t_{i}, S_{i}\right) \text { mit } i=1, \ldots, m \text { und } j=1, \ldots, n
$$

Die Grundgüter des Haushalts $i$ werden mittels einer Kombination verschiedener Inputgüter $x_{i}$ und Zeit $t_{i}$ gemäß der Produktionsfunktion $f_{j, i}$ für das $j$-te Grundgut produziert. $S_{i}$ bezeichnet das „Humankapital“ des Haushalts, welches ebenfalls für die Produktion von $Z_{j, i}$ notwendig ist. Die Idee ist, dass Erfahrung und gewisse Fähigkeiten des Haushalts für die Produktion eines Gutes notwendig sind. Die Nachfrage nach Inputgütern wird, in Anlehnung an die neoklassische Produktionstheorie, als abgeleitete Nachfrage modelliert (Michael \& Becker 1973: 381); die Nachfrage nach Grundgütern führt zu einer abgeleiteten Nachfrage nach Inputgütern (bzw. Produktionsfaktoren). Wenn die Nachfrage nach Grundgütern steigt, dann steigt auch die abgeleitete Nachfrage nach den Produktions- 
faktoren für dieses Gut. Dieser Nachfrageanstieg führt zu einer Preiserhöhung und die durchschnittlichen variablen Kosten des Haushalts steigen. Umgekehrt sinken die Preise der Inputfaktoren sowie die durchschnittlichen variablen Kosten, wenn die Produktion des Grundguts abfällt. Beides führt im Laufe der Zeit zu Verhaltensveränderungen.

Auch wenn die Erklärung von Verhaltensänderungen durch Veränderungen im Anreizsystem auf der Annahme basieren, dass Akteure ihre Kosten und Nutzen kalkulieren, so hat Becker auch immaterielle Kosten und Nutzen berücksichtigt. Dieses erweiterte Verständnis von traditionellen ökonomischen Grundbegriffen ermöglicht es, den Ökonomischen Ansatz auch auf Verhalten außerhalb des Markts anzuwenden. Beispielsweise wird der Begriff des „Schattenpreises" für Güter reserviert, die nicht auf dem Markt getauscht werden und deren Preise sich durch ihre Produktionskosten fixieren lassen. Solche weiten Begriffe haben Becker jedoch auch Kritik eingebracht. Begriffe wie Schattenpreise und immaterieller Nutzen können, wenn zu weit gefasst, arbiträr werden und ihren theoretischen sowie empirischen Wert obsolet machen.

Weil die Annahme der stabilen Präferenzen stark kritisiert wurde, ist dieser Aspekt des Becker'schen Ansatzes zentral. Was sich über die Zeit hinweg und zwischen Akteuren verändert ist die Art und Weise, wie die von Akteuren kontinuierlich präferierten Grundgüter produziert werden. Damit verändern sich auch die Konsumentscheidungen des Akteurs mit Blick auf die Inputgüter, die zur Produktion notwendig sind. Meine Präferenz für Gesundheit ändert sich nicht; meine Nachfrage nach Gütern wie Obst, ökologisch verträglichen Lebensmittel, die Anmeldung in einem Fitnessstudio etc., kurz: die Nachfrage nach Gütern, die meine Gesundheit erhalten, ändert sich jedoch in Abhängigkeit von ihren Marktpreisen sowie meinen Einkommensverhältnissen.

Die Strategie, eine Nutzenfunktion als eine Haushaltsproduktionsfunktion zu formulieren, hat den Vorteil, dass Voraussagen über Verhaltensänderungen auf Basis von antizipierten Verhaltensreaktionen auf veränderte Anreizstrukturen und Restriktionen, wie beispielsweise Preis- und Einkommensänderungen, möglich sind. Zusätzlich argumentiert Becker für seine Haushaltsproduktionsfunktion als eine nützliche Alternative zur traditionellen Nutzenfunktion. Seiner Meinung nach basiert letztere auf der unrealistischen Annahme, dass materielle oder immaterielle Güter direkt in die Nutzenfunktion mit einfließen und ihr Konsum den Nutzen des Akteurs direkt beeinflusst. Aus Beckers Perspektive konsumieren Menschen bestimmte Güter nicht um ihrer selbst willen, sondern nur, weil diese Güter ihnen damit die Produktion von Dingen ermöglichen, die fundamentale Präferenzen befriedigen.

Beckers Ökonomischer Ansatz wurde in allen Sozialwissenschaften angewandt. Becker selbst behandelt ein breites Spektrum an Problemen - von traditionellem 
Konsumentenverhalten über altruistisches Verhalten, Suchtverhalten und Diskriminierung bis hin zu Reproduktionsentscheidungen und Familiengründung (Becker 1976, 1994). Da es sich dabei nicht um traditionell ökonomische Fragestellungen handelt, wurde er als „ökonomischer Imperialist“ kritisiert. Nicht zuletzt durch die enge Zusammenarbeit mit dem Chicagoer Soziologen James Coleman wurde Beckers Ansatz bereits früh in der Soziologie angewandt (siehe Braun 1998; Coleman 1990). Beispielsweise haben Coleman (1987) und Jon Elster (1989a, 1989b) aufbauend auf den klassischen Varianten von Becker sowie der EUT zentrale Weiterentwicklungen dieser vorgenommen, um normkonformes Verhalten innerhalb der RCT abzubilden bzw. zu erklären. Coleman hat dafür theoretische Kernbegriffe in Beckers Ansatz wie beispielsweise „Preis“, „Nutzen“, „Einkommen“ und „Kosten“ erweitert und reinterpretiert. Bei Coleman werden die dem normabweichenden Verhalten folgenden gesellschaftlichen Sanktionen als emotionale Kosten, wie beispielsweise das Erfahren von Scham oder sozialem Ausschluss, erfasst. Daneben wurden auch andere Versuche der theoretischen Einbindung von nicht-monetären Motiven, vom Einfluss sozialer Strukturen wie beispielsweise sozialer Netzwerke, von institutionellen Rahmenbedingungen und von Sozialkapital auf Handlungsbedingungen sowie deren Makroeffekten unternommen (Diekmann \& Voss 2004: 13).

Ein Unterschied zwischen Beckers Ansatz und der EUT sowie der SEUT ist ihr Anwendungsproblem. Letztere werden in der Entscheidungstheorie sowie in der Mikroökonomik vielfach auf Individuen bzw. kleine und homogene Gruppen angewandt. Auch wenn beispielsweise in der Analytischen Soziologie gemäß des MI vom Individuum ausgegangen wird, dienen die Varianten der EUT sowie der SEUT dort eher als theoretischer Ausgangspunkt und Mittel zum Zweck, um kollektive Effekte und Regelmäßigkeiten auf der Makroebene zu erklären und vorauszusagen (Diekmann \& Voss 2004: 21). Bei Beckers Ansatz geht es in erster Linie um die Voraussage von Verhaltensänderungen unter sich verändernden Anreizbedingungen, um auf dieser Basis Politikempfehlungen auszusprechen. Beispielsweise stellt eine Erhöhung der Alkoholsteuer eine Veränderung des Anreizsystems dar, deren Effekt auf das Suchtverhalten man vorhersagen möchte. Im zweiten Schritt kann man auf politischer Ebene beurteilen, ob die Steuereinführung zu den gewünschten Verhaltensänderungen führt bzw. ob die erwarteten Einnahmen realisiert werden.

Auch methodologisch kann der Ökonomische Ansatz nicht als Handlungs. theorie im klassischen Sinn angesehen werden. Er ist intellektuell in der Chicagoer Schule und damit in der neoklassischen Preistheorie zu verorten. Es geht Becker bei dessen Anwendung um die Bestimmungsgrößen von Preisen und, allgemeiner, um Voraussagen von Marktveränderungen mit Verweis auf das Zusammenspiel von Preisen, Einkommen und anderen relevanten ökonomischen Variablen. Becker setzt einen instrumentalistischen Theoriebegriff voraus, definiert 
dabei die Wirtschaftswissenschaften als eine Tool Box und versteht vor diesem Hintergrund seinen Ansatz als die ökonomische „Methode“, mittels derer man sich jedem sozialwissenschaftlichen Problem empirisch nähern kann (Becker 1976, 1993, 2009). Seine Methode ermöglicht ihm einen ökonomischen Zugang zu der sozialen Welt, d. h. Verhaltensänderungen unter variierenden Anreizbedingungen zu betrachten.

Im Gegensatz dazu kann man die EUT, die SEUT sowie die Spieltheorie zwar im ersten Schritt als mathematische Theorie ansehen (z. B. Binmore 1994). Im zweiten Schritt können sie durch die Interpretation ihrer zentralen Begriffe als Entscheidungstheorien anwendbar werden. Becker nimmt zwar an, dass jedem sozialen Phänomen die individuelle Entscheidung zugrunde liegt. Es geht ihm in der ökonomischen Perspektive aber nicht um die Erklärung der individuellen Entscheidung, sondern ganz in der Tradition Milton Friedmans um die Voraussage von stabilen Verhaltensregularitäten von Gruppen auf der Makroebene unter sich verändernden Anreizstrukturen (Becker 1976: 5). Darüber hinaus wird seine Produktionsfunktion ebenfalls auf unterschiedliche Akteure angewandt, vor allem aber auf Haushalte und nicht auf Individuen. Es ist daher nicht eindeutig, inwiefern Becker dem MI verpflichtet ist. In Kombination mit weiteren theoretischen Begriffen erlaubt ihm seine Methode aber Hypothesen zur Erklärung von sozialen Zusammenhängen auf Makroebene abzuleiten und empirisch zu testen.

\section{Die Theorie der offenbarten Präferenzen}

In der Chicagoer Tradition wurde spätestens seit Friedman (1953) das Nutzenmaximierungsprinzip oft mit Verweis auf die „als ob“-Annahme interpretiert. Genauer haben sowohl Friedman als auch Becker das Prinzip mit dem Argument gerechtfertigt, dass man in den Wirtschaftswissenschaften nicht wirklich davon ausgehe, dass Akteure tatsächlich ihren Nutzen maximieren. Vielmehr wird angenommen, dass sich Akteure so verhalten als ob sie ihren Nutzen maximieren. Die Theorie der offenbarten Präferenzen setzt hier an. Die „als ob“-Annahme gründet in einer in den Wirtschaftswissenschaften lange Zeit verbreiteten Skepsis gegenüber der theoretischen Einbindung von Handlungsmotiven. Man wollte die ökonomische Theorie von unbeobachtbaren Entitäten und damit von psychologischen Begriffen wie beispielsweise dem Begriff des Bedürfnisses freihalten, da ökonomische Theorien anwendbar sein und testbare Voraussagen zulassen sollten. Da der Gebrauch von Begriffen von unbeobachtbaren Entitäten metaphysische Annahmen mit sich bringt und die empirische Überprüfbarkeit der Theorien 
erschweren bzw. verhindern würden, sollte auf diese verzichtet werden (z. B. Samuelson 1938).

Darüber hinaus argumentierten Ökonomen wie Becker, dass eine Interpretation der Nutzenmaximierungsannahme als psychologisches Prinzip ad hoc Modifikationen der Theorie sowie ad hoc Handlungserklärungen mit Verweis auf Präferenzänderung zuließe, die man nicht empirisch überprüfen könne. Für die Verwendung des Nutzenmaximierungsprinzips als Beschreibung von Marktverhalten spräche dagegen, dass langfristige Abweichungen vom Nutzenmaximierungsprinzip zu Misserfolgen der abweichenden Akteure auf Märkten führen würden und sich die Akteure damit ohnehin nicht auf dem Markt aufhielten. Genauer wird argumentiert, dass nur diejenigen überhaupt auf Märkten überleben können, die sich zumindest so verhalten, als ob sie ihren Nutzen maximieren. Damit nähere sich das Prinzip einer adäquaten Beschreibung des beobachteten Verhaltens.

Neben dieser evolutionstheoretischen Rechtfertigung haben Ökonomen wie Paul Samuelson Strategien vorgeschlagen, um mit Problemen durch unbeobachtbare mentale Zustände umzugehen. Samuelsons Vorschlag manifestiert sich in der Theorie der offenbarten Präferenzen, die er als Alternative zur traditionellen neoklassischen Haushaltstheorie entwickelte. Ursprünglich war sein Ziel, den Fokus alleine auf die Beschreibung von beobachtbarem Verhalten zu legen. Später wurde die Theorie durch die Zusatzannahme ergänzt, dass beobachtetes Verhalten Rückschlüsse auf die Präferenzordnung des Akteurs und damit auf seine Motivation ermöglicht. Da der Ansatz ebenfalls auf einem Rationalitätsbegriff basiert, kann er als eine Variante der RCT bezeichnet werden. Im Folgenden wird jedoch ebenfalls deutlich, dass dieser sich fundamental von Varianten wie dem Ökonomischen Ansatz, der EUT sowie der SEUT unterscheidet.

Die Geschichte der Theorie der offenbarten Präferenzen ist für ihr Verständnis und ihre Verortung relevant, jedoch komplex. Sie kann daher hier nicht rekonstruiert werden. ${ }^{16}$ Es soll nur kurz erwähnt werden, dass ursprünglich ihr theoretischer Kern das sogenannte schwache Axiom der offenbarten Präferenzen bildete (siehe Samuelson 1938: 65). ${ }^{17}$ Die Nutzenfunktion wird durch eine Verhaltensannahme fixiert, nämlich dass das Verhalten eines rationalen Akteurs konsistent ist (vgl. Kapitel II.1). Wenn das Verhalten eines Akteurs das

\footnotetext{
16 Für Beiträge zur historischen Entwicklung der Theorie siehe Hands (2014). Für eine methodologische Diskussion siehe Wong (2006).

17 Die ursprüngliche Terminologie von Samuelson, dass ein Gut gegenüber einem anderen Gut vorgezogen wird (selected over), wurde später nicht aufgegriffen. Stattdessen hat sich der missverständliche Begriff der „offenbarten Präferenzen“ durchgesetzt, welcher den Zirkulari tätsvorwurf gestützt hat.
} 
schwache Axiom erfüllt, dann kann man sagen, dass der Akteur die beste Alternative wählt, die er sich leisten kann; dass die tatsächlich getroffene Entscheidung gegenüber anderen Entscheidungen vorgezogen werden, die ebenfalls getroffen hätten werden können (Varian 2014: 120f.).

Spätere Versionen basieren auf der Idee, dass die Akteurspräferenzen durch das Verhalten offenbart werden. Es wird angenommen, dass Akteure sich immer gemäß ihren Präferenzen entscheiden und, gegeben bestimmter Preis- und Einkommenskonstellationen, diejenige Option wählen, die sie auch tatsächlich in dieser Situation und bei direktem Vergleich vorziehen. Darüber hinaus wird bei der Theorie offenbarter Präferenzen angenommen, dass Präferenzen über den Beobachtungszeitraum hinweg stabil bleiben, d. h. kurzfristig nicht variieren. Diese Annahme ist bedeutsam, da die Konsistenz von Verhalten nur über die Zeit hinweg überprüft werden kann. Obwohl diese Annahme mit Blick auf lange Zeiträume infrage gestellt wurde, so argumentieren manche Ökonomen, könne sie für kürzere Untersuchungsperioden als plausibel angesehen werden (Varian 2014: 118).

Dennoch muss die Variante ebenfalls mit einem Zirkularitätsvorwurf umgehen. Die Theorie besagt, dass beobachtbares Verhalten die tatsächliche Präferenz offenlegt. Diese offenbarte Präferenz wird wiederum herangezogen, um das Verhalten zu erklären, welches sie offenbart. Diese Erklärung erscheint zirkulär. Um dem Zirkularitätsvorwurf zu begegnen, wird der Begriff der „offenbarten Präferenz" schlicht als das beobachtete Wahlverhalten des Akteurs eines Bündels $x$ interpretiert, obwohl ein anderes Bündel $y$ verfügbar und bezahlbar gewesen wäre. Mit dieser verhaltensbasierten Interpretation von Präferenzen wird versucht die dem Zirkularitätsvorwurf zugrundeliegende Interpretation von Präferenzen als mentale Zustände von sich zu weisen (Varian 2014: 121). Es bleibt jedoch unklar, inwiefern Vertreter dieser Präferenzinterpretation dem Vorwurf tatsächlich entgehen können, falls die Theorie zur Verhaltenserklärung herangezogen werden soll.

Die ursprüngliche Idee der Theorie war, dass alleine durch die plausible Minimalanforderung der temporären Konsistenz von menschlichem Verhalten die Nutzenfunktion des Akteurs ableitbar und damit beobachtbares Verhalten beschreibbar wird. Formal beginnt die traditionelle Variante mit einer postulierten Nachfragefunktion. Damit unterscheidet sie sich von axiomatischen Varianten darin, dass sie nicht im ersten Schritt von einer Präferenzordnung ausgeht. Neben der Schwierigkeit der empirischen Überprüfbarkeit kann diese Variante die Kritik umgehen, dass Akteurspräferenzen nicht die Rationalitätsaxiome der EUT bzw. der SEUT erfüllen. Sie muss jedoch rechtfertigen, warum sich Präferenzen über einen langen Zeitraum nicht ändern - eine Annahme, die ebenfalls vielfach als unplausibel kritisiert wurde. 


\section{I.3.3 Zusätzliche Varianten und Erweiterungen der RCT}

Neben den fünf Spielarten der RCT gibt es noch zahlreiche andere Varianten, die in den Sozialwissenschaften zur Anwendung kommen. Dazu gehören die vor allem in der Politikwissenschaft angewandte und auf Kenneth Arrow (1951) zurückgehende Sozialwahltheorie, die auf Gruppenentscheidungen wie Wahlen oder andere politische Abstimmungen angewandt wird. Dabei geht es primär um das Problem, Aggregationsregeln zu identifizieren, die es erlauben, von verschiedenartigen individuellen Präferenzen auf eine Gruppenpräferenz bzw. -entscheidung zu schließen. Eine weitere Variante ist die sogenannte Public-Choice-Theorie, die auf politische Entscheidungen und Entscheidungsfindungsprozesse von Interessengruppen, politischen Parteien sowie in Verwaltungen des öffentlichen Sektors bzw. innerhalb des Staatsapparats angewandt wird (Downs 1957; Buchanan \& Tullock 1962).

Weniger prominente Varianten sind Gérard Debreus axiomatische Wertheorie (Debreu 1959) sowie die Praxeologie von Ludwig von Mises (1940). Beide werden primär in den Wirtschaftswissenschaften angewandt und sind u. a. auch durch ihre begriffliche und theoretische Eingebundenheit in die jeweiligen theoretischen Ansätze der allgemeinen Gleichgewichtstheorie (im Falle Debreus) sowie der Österreichischen Schule (im Falle von Mises) zu unflexibel, als dass sie sich ohne Weiteres in die Theoriegebäude anderer Sozialwissenschaften integrieren ließen.

Daneben wurden aufgrund der anhaltenden Kritik an den zentralen Begriffen und Annahmen der verschiedenen Varianten einige Erweiterungen unterschiedlicher Ausprägung formuliert. Die breite Anwendung der RCT auf menschliches Verhalten einerseits und das eng gefasste Nutzenmaximierungsprinzip zur theoretischen Beschreibung von Verhalten (je nach Variante) andererseits stellte für Sozialwissenschaften wie beispielsweise die Soziologie eine theoretische Herausforderung dar. Genauer wurde die RCT mit Verweis auf Max Webers Idealtypen-Methodologie oft in direkten Zusammenhang gebracht mit dem Motiv der Zweckrationalität, d. h. der Idee, dass Menschen ihre persönlichen Ziele erreichen wollen und dafür entsprechend die besten, ihnen zur Verfügung stehenden Mittel wählen. Dieser Idealtyp greift nach Weber primär in ökonomischen Kontexten wie dem Markt. In anderen Gesellschaftsbereichen wären andere Idealtypen jedoch sinnvoller. Nach Weber sollten sozialwissenschaftlichen Erklärungen verschiedene Motivationstypen zugrunde liegen, welche die Handlungsgründe in unterschiedlichen Gesellschaftsbereichen erfassen. 
Es sollen hier drei Varianten der soziologischen RCT erwähnt werden, welche begrifflich und theoretisch auf den bereits vorgestellten Varianten basieren, jedoch innerhalb der Soziologie und speziell für die Bedürfnisse der Disziplin entwickelt wurden. Zuerst sei das Modell der Frame-Selektion von Hartmut Esser (2001, 2010) als eine situative Handlungstheorie (Rössel \& Weingärtner 2016) zu nennen (vgl. Kapitel IV.2). Die Grundannahme ist, dass Menschen auf Basis ihrer Wahrnehmung einer Situation entscheiden. Vor dem Handeln erfolgt eine Selektion der Orientierung, die der Entscheidungssituation einen Rahmen gibt, aus dem sich wiederum u. a. Ziele, Mittel, Wahrscheinlichkeiten, Erwartungen und Werte ergeben (Esser 1999). Die Idee ist, dass diese auch in anderen Varianten der RCT verwendeten Variablen durch die Situation beeinflusst werden. Handlungen sind rational, da sie innerhalb eines Frames immer noch zielorientiert sind.

Zweitens sei das kognitivistische Modell von Raymond Boudon (1996) zu nennen. Der zentrale Begriff ist der der kognitiven Rationalität, bei der man im Sinne des Weber'schen Idealtyps der Wertrationalität annimmt, dass ein Akteur ,gute Gründe“ für sein Verhalten hat. Damit gründet eine Entscheidung nicht mehr nur in Kosten-Nutzen-Kalkulationen, sondern wird auf Basis von Anschauungsabwägungen getroffen, je nachdem, was einem Akteur plausibel, wahr oder wahrscheinlich erscheint. Zuletzt sei die Variante von Siegwart Lindenberg (1993) zu nennen, welche durch eine erweiterte Annahmebasis einen Akteur als ,a restricted, resourceful, expecting, evaluating, maximizing man [or woman]“ (RREEMM) beschreibt. Die Kernidee ist, dass Akteure in ihren Handlungsmöglichkeiten eingeschränkt sind, ihre Ressourcen aber gezielt einsetzen können. Ein Akteur gründet seine Überlegungen auf subjektiven Erwartungen, um seine Handlungsoptionen mit Blick auf seine Ziele zu beurteilen. Er entscheidet sich für die Option, die seinen Nutzen maximiert. Ein Ziel dieser differenzierten Variante der SEUT ist der Entwurf eines realistischeren Verhaltensmodells.

Schlussendlich wurde die RCT auch im Rahmen der Verhaltensökonomik erweitert, um Motive wie Normkonformismus, emotionales Verhalten, Altruismus, Fairness oder Reziprozität zu modellieren; die Modelle sozialer Präferenzen sind ein wichtiges Resultat eines solchen Versuchs. Sie basieren hauptsächlich auf Erkenntnissen der experimentellen Entscheidungs- und Spieltheorie, die zeigen, dass Menschen nicht eigennützig motiviert sind bzw. ihre Präferenzen nicht immer und ausschließlich den Rationalitätsaxiomen der EUT sowie der SEUT genügen, sondern andere Eigenschaften aufweisen. Das Diktatorspiel sowie das Ultimatumspiel haben gezeigt, dass Voraussagen auf Basis der Spieltheorie in vielen Zusammenhängen nicht bestätigt werden können (vgl. Kapitel II.4; Kapitel IV.1). 


\section{I.3.4 Schluss}

Die zentralen Spielarten der RCT zeigen, dass die drei dem Received View zugrundeliegenden Merkmale nicht als notwendige Bedingungen gelten können, um einen Ansatz als eine Spielart der RCT einzuordnen. Alle drei Merkmale - (1) eine RCT hat den methodologischen Status einer wissenschaftlichen Theorie, (2) eine RCT geht einher mit einer Verpflichtung zum MI und (3) eine RCT stützt sich auf ein Rationalitätsverständnis, welches menschliches Verhalten als selbstbezogen bzw. egoistisch beschreibt - sind nicht bei allen Varianten gleichermaßen gege ben. Es stellt sich daher die Frage, welche Anforderungen ein theoretischer Ansatz erfüllen muss, um als RC-Variante zu gelten. Im Folgenden werden drei Anforderungen vorgestellt, die allen Varianten zugrunde liegen und damit erlauben, diese als verwandt zu klassifizieren (siehe auch Diekmann \& Voss 2004: 14ff.). Die Anforderungen sind so allgemein, dass sie abhängig von ihren Ausprägungen zu fundamental verschiedenen Varianten führen können. Daher sprechen wir hier von Minimalanforderungen der RCT.

Erstens geht es bei jeder Variante darum, Akteursverhalten zu erfassen. Je nach Spezifikation ist das Ziel dabei Verhalten zu beschreiben, zu erklären, vorauszusagen oder auch vorzuschreiben. Bei Akteuren kann es sich sowohl um Individuen also auch um kollektive Akteure handeln. Zweitens beginnen alle Varianten mit einem Entscheidungsproblem, wobei die Akteurshandlung als Lösung für das Problem aufgefasst wird. Die genaue Form des Entscheidungsproblems kann jedoch variieren. Dessen Darstellung im Falle der SEUT sieht vor, dass Akteure sich zwischen verschiedenen verfügbaren Handlungsalternativen auf Basis ihrer Präferenzen und im Lichte möglicherweise eintretender Umweltereignisse entscheiden. Bei Becker wird angenommen, dass Akteure begrenzte Ressourcen zur Verfügung haben bzw. unter gegebenen Restriktionen handeln, woraus sich die Notwendigkeit einer Allokation dieser Ressourcen ergibt. Es wird außerdem angenommen, dass Akteure bestimmte Ziele verfolgen. Bei Ressourcen ebenso wie bei Restriktionen kann es sich um materielle Elemente handeln wie beispielsweise um verfügbares Einkommen oder Güterpreise. Es können aber ebenso gut auch immaterielle Dinge wie etwa die verfügbare Zeit, existierende Technologien, institutionelle Regelungen sowie rechtliche Einschränkungen berücksichtig werden.

Drittens wird angenommen, dass die Akteure das Entscheidungsproblem mittels einer Entscheidungsregel lösen bzw. sich gemäß dieser Regel entscheiden. Die gängigsten Entscheidungsregeln basieren auf der Idee eines Optimierungskalküls, d. h., dass Akteure im Rahmen ihrer Möglichkeiten ihre gegenwärtige Situation verbessen. Es gibt andere Entscheidungsregeln, die auf die Minimierung des maximalen Schadens, minimales Bedauern, begrenzte Rationalitätskalküle oder auch auf einfache Anspruchserfüllung (satisficing) abzielen. Trotz 
ihrer Unterschiede basieren die meisten Entscheidungsregeln auf der Annahme, dass Akteure bei ihren Entscheidungen vernünftig vorgehen. Wie der Name der RCT sagt, wird „vernünftig“ in der Regel mit „rational“ gleichgesetzt. Anders formuliert haben viele Entscheidungsregeln ihren Ursprung in einem von vielen Sozialwissenschaften akzeptierten methodologischen Postulat, welches vorschreibt, dass menschliches Handeln als rationales Handeln theoretisch gefasst werden soll.

Was der Rationalitätsbegriff im Einzelfall bedeutet, wird bei dem Prinzip des methodologischen Rationalismus offengelassen. Die Definition von rationalem Handeln als eigennütziges Handeln innerhalb des Received Views ist nur eine von verschiedenen Begriffsdefinitionen, die in den verschiedenen Spielarten der RCT Verwendung finden. Dessen Definition kann in Abhängigkeit der Interpretation anderer zentraler Begriffe wie dem Präferenz- oder dem Nutzenbegriff je nach Spielart variieren. In axiomatischen Handlungstheorien wird der Nutzenbegriff weit gefasst, sodass trotz der Definition von rationalem Handeln als nutzenmaximierendes Handeln auch altruistisches sowie normkonformes Handeln erfasst werden kann. Daneben basiert die Theorie der offenbarten Präferenzen auf der Definition von rationalem Handeln als konsistentes Handeln. Bei Becker wird rationales Handeln als nutzenmaximierendes Handeln definiert, wobei die Nutzenmaximierung hier alleine auf der Basis von Kosten-Nutzen-Abwägungen getroffen wird. Der Kosten- sowie der Nutzenbegriff schließen bei Becker immaterielle Kosten und Nutzen aber mit ein.

Alle drei Anforderungen sind so allgemein formuliert, dass sie je nach Variante weiter spezifiziert werden können. Die Spezifizierung hängt davon ab, welche Variante für ein bestimmtes Problem angemessen ist. Darüber hinaus ist die Auswahl der Variante in verschiedenen Disziplinen davon abhängig, welche epistemischen Werte bei der Theoriewahl priorisiert werden. Beispielsweise sind „Einfachheit“ und „Voraussagekraft“ Kernkriterien für die Theoriewahl in den Wirtschaftswissenschaften. In der Soziologie und der Politikwissenschaft hingegen wird auch die „Erklärungskraft“ und die „empirische Adäquatheit“ einer Theorie in den Vordergrund gestellt. Vor diesem Hintergrund ist es wichtig anzuerkennen, dass der Prozess der Spezifizierung in unterschiedlichen Varianten der Theorie enden kann. 


\section{Literatur}

Abraham, M. \& T. Voss, 2002: Contributions of Rational Choice Theory to Modern Sociology: An Overview. S. 136-167 in: N. Genov (Hrsg.), Adavances in Sociological Knowledge Over Half a Century. Paris: International Social Science Council.

Allais, M., 1953: Le comportement de l'homme rationnel devant le risque: critique des postulats et axiomes de l'école Américaine. Econometrica 21: 503-546.

Arrow, K., 1951: Social Choice and Individual Values. New Haven: Yale University Press.

Becker, G.S., 1976: The Economic Approach to Human Behavior. Chicago: University of Chicago Press.

Becker, G.S., 1993: Nobel Lecture: The Economic Way of Looking at Behavior. Journal of Political Economy 101: 385-409.

Becker, G.S., 2009: Gary S. Becker. S. 132-152 in: K.I. Horn (Hrsg.), Roads to Wisdom, Conversations with Ten Nobel Laureates in Economics. Cheltenham: Edward Elgar.

Bicchieri, C., 2006: The Grammar of Society: The Nature and Dynamics of Social Norms. Cambridge: Cambridge University Press.

Binmore, K., 1994: Game Theory and the Social Contract. Volume 1: Playing Fair. Cambridge: MIT Press.

Boudon, R., 1996: The 'Cognitivist Model': A Generalized 'Rational-Choice Model'. Rationality and Society 8: 123-150.

Boudon, R., 2003: Beyond Rational Choice Theory. Annual Review of Sociology 291: 1-21.

Braun, N., 1998: Socially Embedded Exchange. Frankfurt a. M.: Lang.

Braun, N., 2009: Rational Choice Theorie. S. 395-418 in: G. Kneer \& M. Schroer (Hrsg.), Handbuch soziologische Theorien. Wiesbaden: VS Verlag für Sozialwissenschaften.

Buchanan, I.M. \& G. Tullock, 1962: The Calculus of Consent: Logical Foundations of Constitutional Democracy. Michigan: Arbor.

Coleman, J.S., 1987: Norms as Social Capital. S. 133-155 in: G. Radnitzky \& P. Bernholz (Hrsg.), Economic Imperialism: The Economic Method Applied Outside the Field of Economics. New York: Paragon House Publishers.

Coleman, J.S., 1990: Foundations of Social Theory. Cambridge: The Belknap Press of Harvard University Press.

Davidson, D., 1963: Actions, Reasons, and Causes. S. 3-21 in: D. Davidson, Essays on Actions and Events. Oxford: Oxford University Press.

Davidson, D., J.C.C. McKinsey \& P. Suppes, 1955: Outlines of a Formal Theory of Value, 1. Philosophy of Science 22: 140-160.

Debreu, G., 1959: The Theory of Value: An Axiomatic Analysis of Economic Equilibrium. New York: Wiley.

Diekmann, A. \& T. Voss, 2004: Die Theorie rationalen Handelns. Stand und Perspektiven.

S. 13-29 in: A. Diekmann \& T. Voss (Hrsg.), Rational-Choice-Theorie in den Sozialwissenschaften. Anwendungen und Probleme. München: Oldenbourg.

Diekmann, A. \& T. Voss, 2018: Rational Choice-Rezeption in der deutschsprachigen Soziologie. S. 662-682 in: S. Moebius \& A. Ploder (Hrsg.), Handbuch Geschichte der deutschsprachigen Soziologie. Band 1: Geschichte der Soziologie im deutschsprachigen Raum. Wiesbaden: Springer VS.

Dietrich, F. \& C. List, 2013: A Reason-Based Theory of Rational Choice. Noûs 47: 104-134.

Downs, A., 1957: An Economic Theory of Democracy. New York: Harper \& Row. 
Edgeworth, F.Y., 1881: Mathematical Psychics: An Essay on the Application of Mathematics to the Moral Sciences. London: C. Kegan Paul \& Co.

Ellsberg, D., 1961: Risk, Ambiguity, and the Savage Axioms. The Quarterly Journal of Economics 75: 643-669.

Elster, J., 1985: Making Sense of Marx. Cambridge: Cambridge University Press.

Elster, J., 1989a: The Cement of Society: A Study of Social Order. Cambridge: Cambridge University Press.

Elster, J., 1989b: Social Norms and Economic Theory. Journal of Economic Perspectives 3: 99-117.

Esser, H. 1999. Soziologie. Spezielle Grundlagen. Band 1: Situationslogik und Handeln. Frankfurt a. M.: Campus.

Esser, H. 2001. Soziologie. Spezielle Grundlagen. Band 6: Sinn und Kultur. Frankfurt a. M.: Campus.

Esser, H. 2010. Das Modell der Frame-Selektion. Eine allgemeine Handlungstheorie für die Sozialwissenschaften? S. 45-62 in: G. Albert \& S. Sigmund (Hrsg.), Soziologische Theorie kontrovers. Sonderheft 50 der Kölner Zeitschrift für Soziologie und Sozialpsychologie. Wiesbaden: VS Verlag für Sozialwissenschaften.

Friedman, M., 1953: The Methodology of Positive Economics. S. 3-43 in: M. Friedman, Essays in Positive Economics. Chicago: University of Chicago Press.

Guala, F., 2019: Preferences: Neither Behavioural nor Mental. Economics \& Philosophy 35: 383-401.

Hands, W., 2014: Paul Samuelson and Revealed Preference Theory. History of Political Economy 46: 85-116.

Hedström, P. \& P. Ylikoski, 2010: Causal Mechanisms in the Social Sciences. Annual Review of Sociology 36: 49-67.

Herfeld, C.S., 2012: The Potentials and Limitations of Rational Choice Theory: An Interview with Gary Becker. Erasmus Journal for Philosophy and Economics 5: 73-86.

Herfeld, C.S., 2013: The Many Faces of Rational Choice Theory. Witten: Witten/Herdecke University.

Herfeld, C.S., 2020: The Diversity of Rational Choice Theory: A Review Note. Topoi: $10.1007 /$ s11245-018-9588-7.

Jevons, W., [1871] 1888: The Theory of Political Economy. 3. Aufl., London: Macmillan and Co. Knight, F.H., 1921: Risk, Uncertainty, and Profit. Boston: Hart, Schaffner and Marx.

Lindenberg, S., 1993: Framing, Empirical Evidence, and Applications. S. 11-38 in: P. HerderDorneich, K.-E. Schenk \& D. Schmidtchen (Hrsg.), Jahrbuch für Neue Politische Ökonomie. Band 12: Neue Politische Ökonomie von Normen und Institutionen. Tübingen: Mohr.

Machina, M.J., 1989: Dynamic Consistency and Non-Expected Utility Models of Choice under Uncertainty. Journal of Economic Literature 27: 1622-1668.

Mantzavinos, C., 2009: A Note on Methodological Individualism. S. 211-215 in: M. Cherkaoui \& P. Hamilton (Hrsg.), Raymond Boudon. A Life in Sociology. Oxford: Bardwell Press.

Mehta, I., C.S. Starmer \& R. Sugden, 1994: The Nature of Salience: An Experimental Investigation of Pure Coordination Games. American Economic Review 84: 658-673.

Michael, R.T. \& G.S. Becker, 1973: On the New Theory of Consumer Behavior. The Swedish Journal of Economics 75: 378-396.

Milton, F., 1953: Essays in Positive Economics. Chicago: University of Chicago Press.

Nash, J., 1950: Equilibrium Points in n-Person Games. Proceedings of the National Academy of Science 36: 48-49. 
Opp, K.-D., 1999: Contending Conceptions of the Theory of Rational Action. Journal of Theoretical Politics 11: 171-202.

Opp, K.-D., 2014: Methodologie der Sozialwissenschaften. Wiesbaden: VS Verlag für Sozialwissenschaften.

Opp, K.-D. 2019: Die Theorie rationalen Handelns, das Modell der Frame-Selektion und die Wirkungen von Bestrafungen auf Kooperation. Zeitschrift für Soziologie 48: 97-115. Ramsey, F.P., 1928: Truth and Probability. S. 156-198 in: R.B. Braithwaite (Hrsg.), The Foundations of Mathematics and Other Logical Essays. London: Routledge \& Kegan Paul. Raub, W., 2017: Rational Models. Utrecht: Utrecht University.

Rosenberg, A., 2015: Philosophy of Social Science. Boulder: Westview.

Rössel, J. \& S. Weingartner, 2016: Rational Choice-Theorie in der Kultursoziologie. S. 1-19

in: S. Moebius, F. Nungesser \& K. Scherke (Hrsg.): Handbuch Kultursoziologie. Band 2:

Theorien - Methoden - Felder. Wiesbaden: VS Verlag für Sozialwissenschaften. Theorien - Methoden - Felder. Wiesbaden: VS Verlag für Sozialwissenschaften Economica
Samuelson, P. A., 1938: A Note on the Pure Theory of Consumer's Behaviour. Econo
5: 61-71.

Satz, D. \& J. Ferejohn, 1994: Rational Choice and Social Theory. Journal of Philosophy 91: 71-87.

Savage, L.J., 1954: The Foundations of Statistics. New York: Dover.

Schelling, T.C., 1960: The Strategy of Conflict. Cambridge: Harvard University Press.

Schotter, A., 2006: Strong and Wrong: The Use of Rational Choice Theory in Experimental

Economics. Journal of Theoretical Politics 18: 498-511.

Steele, K., 2014: Choice Models. S. 185-208 in: N. Cartwright \& E. Montuschi (Hrsg.),

Sugden, R., 2016: Ontology, Methodological Individualism, and the Foundations of the Social Sciences. Journal of Economic Literature 54: 1377-1389.

Thoma, J., 2019: Decision Theory. S. 57-106 in: R. Pettigrew \& J. Weisberg (Hrsg.), The Open Handbook of Formal Epistemology. PhilPapers Foundation. Routledge.

Vanderschraaf, P. \& G. Sillari, 2014: Common Knowledge. The Stanford Encyclopedia of erschraaf, P. \& G. Sillari, 2014: Common Knowledge. The Stanford Encyclopedia of ${ }^{2}$ Phe
Philosophy: https://plato.stanford.edu/archives/spr2014/entries/common-knowledg
(abgerufen am 03. 03.2020).

Varian, H.R., 2014: Intermediate Microeconomics: A Modern Approach. New York: Norton. Varian, H.R., 2014: Intermediate Microeconomics: A Modern Approach. New York: Nor
von Mises, L., 1940: Human Action: A Treatise on Economics. 4. Aufl., Irvington-on-Hudson
Foundation for Economic Education, Inc. Foundation for Economic Education, Inc.
veumann, I., 1928: Zur Theorie der Gesellschaftsspiele. Mathematische Annalen 100: von Neumann, I. \& O. Morgenstern, [1944] 1953: Theory of Games and Economic Behavior.
Princeton: Princeton University Press. Vriend, N.J., 1996: Rational Behavior and Economic Theory. Journal of Economic Behavior
Organization 29: 263-85.

Wong, S., 2006: Foundations of Paul Samuelson's Revealed Preference Theory. A Study by the Method of Rational Reconstruction. Oxon: Routledge. 